\title{
Technical note: Validation of a system for monitoring rumination in dairy cows
}

\author{
K. Schirmann, ${ }^{*} \dagger$ M. A. G. von Keyserlingk, ${ }^{*}$ D. M. Weary, ${ }^{*}$ D. M. Veira, $\ddagger$ and W. Heuwieser ${ }^{*} \dagger^{1}$ \\ *Animal Welfare Program, Faculty of Land and Food Systems, The University of British Columbia, 2357 Main Mall, Vancouver, V6T 1Z4, Canada \\ †Department of Veterinary Medicine, Clinic for Animal Reproduction, Freie Universität Berlin, 14163 Berlin, Germany \\ $\ddagger$ Pacific Agri-Food Research Centre, Agriculture and Agri-Food Canada, PO Box 1000, Agassiz, British Columbia, V0M 1A0, Canada
}

\section{ABSTRACT}

Increased rumination in dairy cattle has been associated with increased saliva production and improved rumen health. Most estimates of rumination are based on direct visual observations. Recently, an electronic system was developed that allows for automated monitoring of rumination in cattle. The objective was to validate the data generated by this electronic (Hi-Tag, SCR Engineers Ltd., Netanya, Israel) rumination monitoring system. Assessments of 2 independent observers were highly correlated $(\mathrm{r}=0.99, \mathrm{n}=23)$, indicating that direct human observations were suitable as the reference method. Measures from the Hi-Tag electronic system were validated by comparing values with those from a human observer for fifty-one 2-h observation periods from 27 Holstein cows. Rumination times (35.1 $\pm 3.2 \mathrm{~min}$ ) from the electronic system were highly correlated with those from direct observation $\left(r=0.93, \mathrm{R}^{2}\right.$ $=0.87, \mathrm{n}=51$ ), indicating that the electronic system was an accurate tool for monitoring this behavior in dairy cows.

Key words: rumination, feeding behavior, dairy cow, validation

Rumination is the process of regurgitation, remastication, salivation, and swallowing of ingesta to reduce the particle size of feedstuffs and enhance fiber digestion (Welch, 1982; Beauchemin, 1991). Although most rumination takes place while cows are lying down, rumination also takes place concurrently with activities such as standing, walking, scratching, urinating, defecating, and nursing (Beauchemin, 1991). Increased rumination time was associated with increased saliva production and improved rumen health (Beauchemin, 1991), because of the saliva carrying buffer for the ruminal $\mathrm{pH}$. Decreased rumination is interpreted as an indicator of stress (Herskin et al., 2004), anxiety (Bristow and Holmes, 2007), or disease (Welch, 1982; Hansen et al.,

Received May 7, 2009.

Accepted August 26, 2009.

${ }^{1}$ Corresponding author: wolfgang.heuwieser@ubc.ca
2003). Rumination is influenced by nutritional factors such as digestibility of the feed, NDF intake, dietary composition, and forage quality (Welch and Smith, 1970; Beauchemin, 1991).

Rumination is typically monitored through visual observation taken either live (Krause et al., 1998; Couderc et al., 2006) or from video (Lindström et al., 2001). Visual observation is labor intensive, and typically only a few cows can be monitored simultaneously. Indirect methods of monitoring rumination were based on jaw-motion-detecting devices (i.e., strain or pressure gauges) attached to or built into a halter (Luginbuhl et al., 1987; Matsui and Okubo, 1991; Dado and Allen, 1993). These methods provided useful information, but the equipment was cumbersome and had several technical limitations. Most devices required full head halters that included moveable devices located under the jaw. These devices may be uncomfortable for the animals and may have affected their behavior, but numerous studies have shown that they were effective in differentiating jaw movements associated with chewing and ruminating behavior (Beauchemin et al., 1989; Matsui and Okubo, 1991; Dado and Allen, 1993). Early versions of these devices required cables connected to a computer for data transmission and were limited to use on cows housed in tie stalls (Luginbuhl et al., 1987; Beauchemin et al., 1989). Later work described devices developed for use in free-ranging or loose-housed cattle (Matsui, 1994; Rutter et al., 1997; Kononoff et al., 2002). Memory capacities for data storage were limited and halter removal was required to download the data. These technical challenges limited the collection of continuous ( $>21 \mathrm{~d}$ ) rumination data from loose-housed cows (Matsui, 1994).

The Hi-Tag rumination monitoring system (SCR Engineers Ltd., Netanya, Israel) provides output data for rumination time, intervals between regurgitation of boluses, and chewing rate. The system consists of rumination loggers, stationary or mobile readers, and software for processing the electronic records (Data Flow software, SCR Engineers Ltd.). A neck collar positions the logger on the left side of the neck. Re- 
gurgitation and rumination produce distinctive sounds that are recorded by a microphone, processed, and digitally stored. Data are calculated and summarized in 2-h intervals and stored in the memory of the logger for up to $22 \mathrm{~h}$. Unfortunately, the technology allows only for the collection of eleven 2-h intervals $(22 \mathrm{~h})$, after which the first interval recorded is overwritten. In situations in which the data have not been downloaded, results are lost data. Data can be downloaded via readers positioned at locations within the barn (e.g., above the water trough or in the milking parlor) or by a handheld reader. This technology has not been validated for measuring rumination in dairy cattle. Thus, the overall objective was to validate the measures generated by the Hi-Tag system by comparing estimates from the system with those from direct observations. Direct observations may be prone to error, so a secondary aim was to compare rumination data collected by 2 trained investigators via direct human observation to determine interobserver variability. The Hi-Tag software processes the raw data in ways that are not fully transparent. Thus, a final objective was to compare the raw data from the Hi-Tag system with the data output generated by the system software.

This study used 47 Holstein cows from The University of British Columbia Dairy Education and Research Centre (Agassiz, British Columbia, Canada), managed according to the guidelines set by the Canadian Council on Animal Care (1993). Cows, depending on stage of lactation, were fed a TMR formulated according to the recommendations provided by NRC (2001): 1) faroff cows received $43.6 \%$ grass silage, $39.7 \%$ corn silage, $12.7 \%$ straw, and $4.0 \%$ mineral and concentrate mix; 2 ) close-up cows received $43.9 \%$ corn silage, $34.7 \%$ alfalfa hay, and $21.4 \%$ prelactation concentrate and mineral mix on a DM basis (DM: $47.3 \pm 2.8 \%$; CP: $16.4 \pm 0.4 \%$ of DM; ADF: $32.6 \pm 0.9 \%$ of DM; NDF: $44.0 \pm 1.3 \%$ of $\mathrm{DM}$; and $\mathrm{NE}_{\mathrm{L}}: 1.4 \mathrm{Mcal} / \mathrm{kg}$ ); and 3) mid-lactation cows received $39.7 \%$ concentrate and mineral mix, $32.9 \%$ grass silage, $19.2 \%$ corn silage, and $8.2 \%$ alfalfa hay on a DM basis (DM: $47.9 \pm 2.9 \%$; CP: $17.7 \pm 0.5 \%$ of DM; ADF: $22.5 \pm 0.6 \%$ of DM; NDF: $36.1 \pm 1.03 \%$ of DM; and $\mathrm{NE}_{\mathrm{L}}: 1.5 \mathrm{Mcal} / \mathrm{kg}$ ).

Before the live observations began for the trials, the 2 observers were trained in the following manner. First, based on information gathered from the scientific literature a draft definition of rumination was written. The 2 observers undertook live observations using the draft definition and when disagreement occurred they reviewed the definition until such time that agreement was reached (for final definition see next paragraph). Both observers were then equipped with a laptop computer. The stopwatch option in Excel (version 2003; Microsoft, Redmond, WA) was programmed to record the rumination time. For live visual observations, the 2 observers were positioned outside the observation pen but with an unobstructed view of the individual cow housed in the pen. The observers did not communicate with each other and were unable to view the other's computer display.

The onset of rumination was defined as the time when regurgitation took place, namely when a bolus came up the esophagus and reached the mouth. Observers recorded the start time when a bolus reached the mouth and the cow began to chew rhythmically and recorded the ending time when the bolus was swallowed. With the beginning and end time of every single bolus being recorded, the elapsed time between successive regurgitation events (i.e., the time from the beginning of one rumination event until the beginning of the next), the length of a rumination bout, and the elapsed time between one bolus being swallowed and the following bolus being regurgitated were calculated.

The rumination loggers included a microphone, a microprocessor, and a transponder that were encapsulated into a plastic casing ( size $=100 \mathrm{~mm} \times 80 \mathrm{~mm} \times$ $35 \mathrm{~mm}$; weight $=120 \mathrm{~g}$ ) and fitted onto a nylon collar. The collar consisted of an adjustable nylon strap fitted with a weight $($ size $=70 \mathrm{~mm} \times 70 \mathrm{~mm} \times 30 \mathrm{~mm}$; weight $=540 \mathrm{~g}$ ) that hung below the neck to ensure that the rumination logger retained its position on the left side of the neck. The collar including all components weighed $920 \mathrm{~g}$. Placement of the rumination logger was approximately $20 \mathrm{~cm}$ behind the left ear and 5 to $10 \mathrm{~cm}$ down the left side of the neck.

The Hi-Tag system uses sounds recorded by a builtin microphone to identify regurgitation and rumination. The beginning of a rumination event, as defined by the software, occurred when the system detected the sound associated with regurgitation. As boluses are typically masticated for 30 to $60 \mathrm{~s}$ (Beauchemin, 1991), the algorithm considered rumination events separate if successive regurgitations were separated by at least 30 s.

Data were collected in 3 trials conducted in July and August 2008 (trials 1 and 2, respectively) and in October 2008 (trial 3). During trials 1 and 2, cows were housed individually in open pens $(450 \mathrm{~cm} \times 330 \mathrm{~cm})$ bedded with washed river sand and a layer of fresh straw. Pens were cleaned daily. Fresh water was available ad libitum. Cows were fed ad libitum with fresh feed delivered once daily at approximately $0800 \mathrm{~h}$. In trial 1, 15 cows ( 5 close-up, 5 far-off, 5 fresh cows) were observed for 1 observation period that lasted $2 \mathrm{~h}$. Cows were moved from the freestall barn to the observation pen $2 \mathrm{~h}$ before the beginning of the observation period starting at $1000 \mathrm{~h} \pm 1 \mathrm{~h}$. Six rumination loggers were used. 


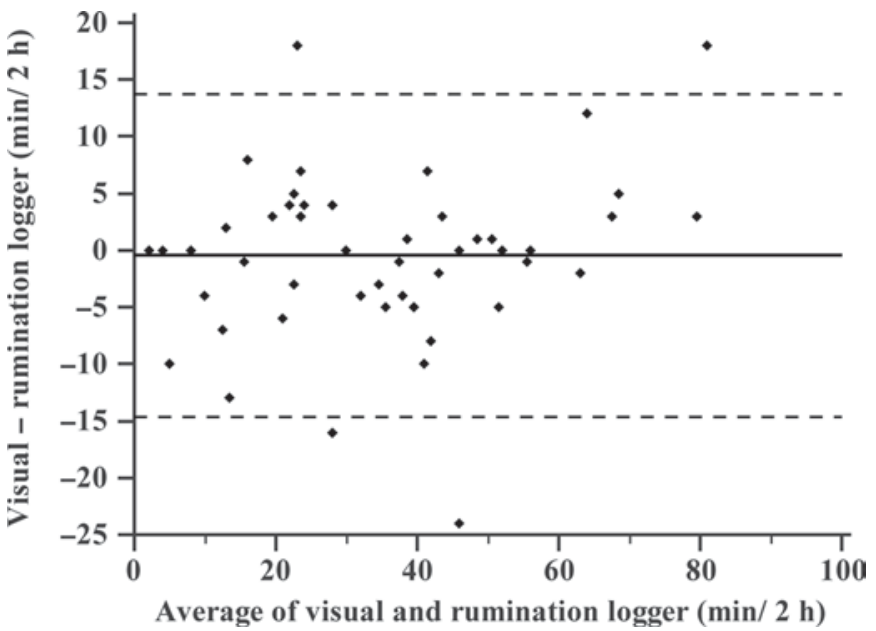

Figure 1. Differences between rumination times assessed by the Hi-Tag rumination loggers (SCR Engineers Ltd., Netanya, Israel) and by direct visual observations (visual - rumination logger) versus the mean of both estimates. Data are shown for fifty-one 2-h periods from 27 cows recorded in trials 1 and 2 (mean difference $=-0.45,95 \%$ confidence interval: -14.7 to 13.8$)$.

In trial 2, 12 cows (9 multiparous, 3 primiparous) 14 $\mathrm{d} \pm 5 \mathrm{~d}$ (mean $\pm \mathrm{SD})$ before actual calving date were used. Cows were fed the close-up TMR as described above. Each of the 12 cows was observed for 3 observation periods that each lasted $2 \mathrm{~h}$. Each cow was fitted with a different rumination logger. Cows were moved to the observation pen at $1800 \pm 1 \mathrm{~h}$. The direct observation periods began at $2000 \pm 1 \mathrm{~h}, 0900 \pm 2 \mathrm{~h}$, and 1100 $\pm 2 \mathrm{~h}$.

Trial 3 used 20 of 24 mid-lactating cows, housed in groups of 12 in 2 pens with 24 deep-bedded, washed river sand stalls. In each pen, 10 of the 12 cows housed were fitted with a rumination logger as described previously. All cows were fed the mid-lactation TMR as described above, fed twice daily at approximately 0700 and $1600 \mathrm{~h}$, and milked twice daily just before delivery of fresh feed.

To determine the inter-investigator reliability, 2 observers independently recorded rumination behavior for 23 observational periods from 10 cows in trial 1 . For the inter-investigator reliability, the correlation coefficient (Pearson) was calculated (SAS Institute, 2003)

The relationship between rumination time estimated by the loggers (dependent variable) and the estimate from direct observation (independent variable) was assessed using Pearson correlation coefficient and linear regression (SAS Institute, 2003).

The agreement between the visual observation measure and the measure generated by the rumination logger was assessed for the total time spent ruminating within the observed 2-h period using linear regression and the Bland and Altman (1986) method (Figure 1) to visualize the distribution of the residuals. The Bland and Altman method involved plotting the mean of the paired measurements (x-axis) obtained by direct visual observations and via Hi-Tag rumination loggers against their difference (y-axis). Figure 1 illustrates variation of the residuals in relationship to total rumination time for the same 2-h interval. The $95 \%$ confidence interval around the mean of the differences was calculated and superimposed on the plot.

The rumination times estimated by the 2 independent investigators were highly correlated $(\mathrm{r}=0.99, \mathrm{n}=$ $23 ; P<0.001$ ). Average inter-investigator variation was $1.1 \%$; the maximum variation between measures for a single observation was $10.5 \%$. These results demonstrate that rumination times can be assessed reliably through direct observation. Rutter et al. (1997) reported high concordance (92.9\%) between 2 observers' estimates of rumination times.

The Hi-Tag rumination monitoring system provided estimates of rumination time (35.1 $\mathrm{min} \pm 3.2 \mathrm{~min})$ that were similar to direct observation (34.7 $\mathrm{min} \pm 20.3$ $\min$ ). For trials 1 and 2, correlation coefficients (r) and coefficients of determination $\left(\mathrm{R}^{2}\right)$ between rumination times measured electronically with the rumination logger and by direct observation were high (trial 1: $\mathrm{r}=$ $0.96, \mathrm{R}^{2}=0.93, \mathrm{n}=15, P<0.001 ;$ trial $2: \mathrm{r}=0.92$, $\left.\mathrm{R}^{2}=0.86, \mathrm{n}=36, P<0.001\right)$. Plotting the difference between estimates generated by the rumination loggers and direct visual observation against the mean of the 2 estimates showed that disagreements between the methods were evenly distributed across the range (Figure 1). The mean difference of -0.45 showed that the rumination loggers reported rumination times that were 0.45 min longer than the values determined by visual observation during a 2 -h observation period. The 95\% CI (lower and upper limits of agreement) for the point estimates of the mean difference were 13.8 and -14.7 min. By using the Bland and Altman (1986) method, approximately $95 \%$ of data points were within the upper and lower limits of agreement. Overall variation between estimates averaged $6.1 \%$; the maximum variation between estimates for a single observation in a single case was $96.3 \%$, with the observer detecting only $7 \mathrm{~min}$ of rumination and the logger recording 20 min. Methodological issues such as a loose or incorrectly positioned collar may have contributed to this variation. Errors associated with the visual observation method such as accurately detecting the start and finish of each rumination bout or in discriminating jaw movements as eating versus ruminating, particularly when the head of the cow was in the feed bin, may 
have contributed to this variance. Previous validation experiments assessing jaw movement loggers reported comparable correlations $(\mathrm{r}=0.91$ to 0.98 ; Beauchemin et al., 1989) and overall variance $(9.2 \%$; Kononoff et al., 2002) for daily rumination time obtained by loggers and direct observation.

Results from trial 3 (20 cows, each followed for 3 d) were used to determine correlation coefficients for rumination time per $24 \mathrm{~h}$ between raw data obtained from the rumination loggers (twelve 2-h cells) and daily rumination time as processed by the Data Flow software. Correlation coefficients were high $(\mathrm{r}=0.96, \mathrm{n}$ $=60, P<0.001)$. Moreover, differences between the 2 calculations of daily rumination time per cow (raw data from the logger: $526.2 \mathrm{~min} \pm 5.3 \mathrm{~min}$, Data Flow software: $516.2 \mathrm{~min} \pm 5.6 \mathrm{~min})$ were small $(10 \pm 37$ $\mathrm{min} / 24 \mathrm{~h}$ ). These data demonstrate that the Data Flow software can be used to summarize 24-h data of rumination time.

The monitoring of daily rumination time as well as the time spent ruminating within 2-h intervals throughout the day could be useful for research as well as for commercial purposes (e.g., for detecting cows close to parturition or sick cows), but further research is required.

Although the Hi-Tag rumination monitoring system has been commercially available since 2007 , this is the first validation of the system to our knowledge. Our data provide evidence that the Hi-Tag logger, although imperfect, does provide a reasonable measure of rumination time and can be a useful tool in research and commercial applications.

\section{ACKNOWLEDGMENTS}

We thank the staff of The University of British Columbia Dairy Education and Research Centre and the University's Animal Welfare Program. In particular, we thank Heather Afford for her help with the visual observations. Kathrin Schirmann was funded by a scholarship from Tiergyn Berlin e.V. This project was funded by the Natural Sciences and Engineering Research Council of Canada, Freie Universität Berlin, and Dairy Farmers of Canada and many other donors listed on the Animal Welfare Web site at http://www. landfood.ubc.ca/animalwelfare.

\section{REFERENCES}

Beauchemin, K. A. 1991. Ingestion and mastication of feed by dairy cattle. Vet. Clin. North Am. Food Anim. Pract. 7:439-462.

Beauchemin, K. A., S. Zelin, D. Genner, and J. G. Buchanan-Smith. 1989. An automatic system for quantification of eating and ruminating activities of dairy cattle housed in stalls. J. Dairy Sci. $72: 2746-2759$.

Bland, J. M., and D. G. Altman. 1986. Statistical methods for assessing agreement between two methods of clinical measurement. Lancet $338: 307-310$.

Bristow, D. J., and D. S. Holmes. 2007. Cortisol levels and anxietyrelated behaviors in cattle. Physiol. Behav. 90:626-628.

Canadian Council on Animal Care. 1993. Guide to the Care and Use of Experimental Animals. Vol.1. E. D. Olfert, B. M. Cross, and A. A. McWilliam, ed. CCAC. Ottawa, Ontario, Canada.

Couderc, J. J., D. H. Rearte, G. F. Schroeder, J. I. Ronchi, and F. J. Santini. 2006. Silage chop length and hay supplementation on milk yield, chewing activity, and ruminal digestion by dairy cows. J. Dairy Sci. 89:3599-3608.

Dado, R. G., and M. S. Allen. 1993. Continuous computer acquisition of feed and water intakes, chewing, reticular motility, and ruminal $\mathrm{pH}$ of cattle. J. Dairy Sci. 76:1589-1600.

Hansen, S. S., P. Norgaard, C. Pedersen, R. J. Jorgensen, L. S. B. Mellau, and J. D. Enemark. 2003. The effect of subclinical hypocalcaemia induced by $\mathrm{Na}_{2}$ EDTA on the feed intake and chewing activity of dairy cows. Vet. Res. Commun. 27:193-205.

Herskin, M. S., L. Munksgaard, and J. Ladewig. 2004. Effects of acute stressors on nociception, adrenocortical responses and behavior of dairy cows. Physiol. Behav. 83:411-420.

Kononoff, P. J. H. A. Lehman, and A. J. Heinrichs. 2002. Technical note: A comparison of methods used to measure eating and ruminating activity in confined dairy cattle. J. Dairy Sci. 85:1801-1803.

Krause, M., K. A. Beauchemin, L. M. Rode, B. I. Farr, and P. Norgaard. 1998. Fibrolytic enzyme treatment of barley grain and source of forage in high-grain diets fed to growing cattle. J. Anim. Sci. 76:2912-2920.

Lindström, T., I. Redbo, and K. Uvnäs-Moberg. 2001. Plasma oxytocin and cortisol concentrations in dairy cows in relation to feeding duration and rumen fill. Physiol. Behav. 72:73-81.

Luginbuhl, J.-M., K. R. Pond, J. C. Russ, and J. C. Burns. 1987. A simple electronic device and computer interface system for monitoring chewing behavior of stall-fed ruminant animals. J. Dairy Sci. 70:1307-1312.

Matsui, K. 1994. A new ambulatory data logger for a long term determination of grazing and rumination behaviour on free-ranging cattle, sheep and goats. Appl. Anim. Behav. Sci. 39:123-140.

Matsui, K., and T. Okubo. 1991. A method for quantification of jaw movements suitable for use on free ranging cattle. Appl. Anim. Behav. Sci. 32:107-116.

NRC. 2001. Nutrient Requirements of Dairy Cattle. 7th rev. ed. National Academy Press, Washington, DC.

Rutter, S. M., R. A. Champion, and P. D. Penning. 1997. An automatic system to record foraging behaviour in free-ranging ruminants. Appl. Anim. Behav. Sci. 54:185-195.

SAS Institute. 2003. SAS User's Guide. Version 9.1. SAS Institute Inc., Cary, NC.

Welch, J. G. 1982. Rumination, particle size and passage from the rumen. J. Anim. Sci. 54:885-894.

Welch, J. G., and A. M. Smith. 1970. Forage quality and rumination time in cattle. J. Dairy Sci. 53:797-800. 